\title{
O caso da Rede Universitária de Telemedicina: análise da entrada da telessaúde na agenda política brasileira
}

| ${ }^{1}$ Angélica Baptista Silva, ${ }^{2}$ |lara Hammerli Sozzi de Moraes |

Resumo: Estudo de abordagem qualitativa sobre a concepção da política de telessaúde no Brasil baseado em modelos de avaliação em saúde e na análise de políticas públicas, mais especificamente na proposta analítica de múltiplos fluxos e no modelo lógico de avaliação de programas em saúde. As fontes de dados consistiram em documentos relacionados à criação da Rede Universitária de Telemedicina (Rute), artigos disponíveis na literatura sobre telessaúde e avaliação em saúde, atas e participações em reuniões de variados fóruns. $\mathrm{O}$ modelo de múltiplos fluxos se concentra nas fases iniciais do ciclo da política pública: a construção da agenda governamental e a escolha de alternativas de políticas. Buscou-se delinear a composição da Rute e responder quais condicionantes levaram os gestores federais de diversos setores a reconhecer a qualidade do sistema como problema na saúde pública e a escolha da implantação de programas de telessaúde como uma das alternativas para a solução. Os processos analisados demonstraram que, na convergência do fluxo de problemas com ambiente político favorável, abriu-se uma janela de oportunidade para a implantação da telessaúde como alternativa vir a ascender como política na agenda de decisão governamental. Porém, para isso, urge a necessidade de inclusão digital no território e identificar as tecnologias da informação e comunicação como inovação para o SUS.

\footnotetext{
> Palavras-chave: telessaúde; política pública; avaliação.
}

\author{
1 Departamento de Ciências \\ Sociais da Escola Nacional de \\ Saúde Pública Sergio Arouca \\ (ENSP/Fiocruz); responsável pela \\ Área de Telessaúde do Instituto \\ de Saúde da Mulher, da Criança \\ e do Adolescente Fernandes \\ Figueira (IFF/Fiocruz). Endereço \\ eletrônico: angelica@fiocruz.br \\ 2 Pesquisadora Titular do \\ Departamento de Ciências \\ Sociais da ENSP/Fiocruz. \\ Endereço eletrônico: ilara@ \\ ensp.fiocruz.br
}


Telessaúde é um conceito polissêmico e recente (NOORANI, 2001; BASHSHUR et al., 2011), que pode ser compreendido, na cibercultura, como um conjunto de fenômenos promovido pelo avanço das telemáticas, seus maquinismos e respectivas redes sociotécnicas participativas (FELINTO, 2006; RÜDIGER, 2011). Partimos do princípio de que telessaúde é toda atividade em rede mediada por computação que promove o intercâmbio entre pesquisa translacional ${ }^{1}$ e serviço de saúde, e que envolve não só o campo de estudos da cibercultura, mas também o da política pública em saúde.

Para analisar telessaúde como política pública, faz-se necessário contextualizar o binômio inclusão/exclusão digital. O termo inclusão digital geralmente é empregado em contextos de elaboração de políticas públicas estruturais e compensatórias de combate à pobreza. Já a exclusão é utilizada em diagnósticos de realidades específicas. Ambos, porém, dizem respeito ao mesmo processo (SILVA, 2005). Nesse sentido, uma singularidade brasileira é que a disseminação das Tecnologias da Informação e da Comunicação (TIC) no território (quadro 1), em especial no Setor Saúde (MAGALHÃES, 2010), não corresponde ao investimento necessário para implantar a telessaúde no Sistema Único de Saúde (SUS). Essa falta de infraestrutura para estabelecer serviços de telessaúde fortalece o que Beck (1999) nomeia como "nova estratificação social da globalização": produz ricos globalizados (com acesso irrestrito a todo tipo de cuidado) e pobres localizados (sem acesso a serviços de saúde essenciais).

Com a Constituição de 1988, o Estado assume o compromisso de garantir saúde para todos, mediante políticas sociais e econômicas voltadas para "o acesso universal e igualitário a ações e serviços para sua promoção, proteção e recuperação" e para a "redução do risco de doença e de outros agravos", (BRASIL 1988, art. 196), criando o SUS.

O conceito de integralidade é expresso no Texto Constitucional como uma das diretrizes do SUS, quando enfatiza o "atendimento integral, com prioridade para as atividades preventivas, sem prejuízo dos serviços assistenciais” (BRASIL 1988, art. 198). Integralidade esta entendida principalmente como ampliação do horizonte de intervenção sobre problemas (MATTOS, 2006), cabendo destacar o acesso às técnicas de diagnóstico e tratamento específicas para cada caso, 

por área urbana e rural em 2010

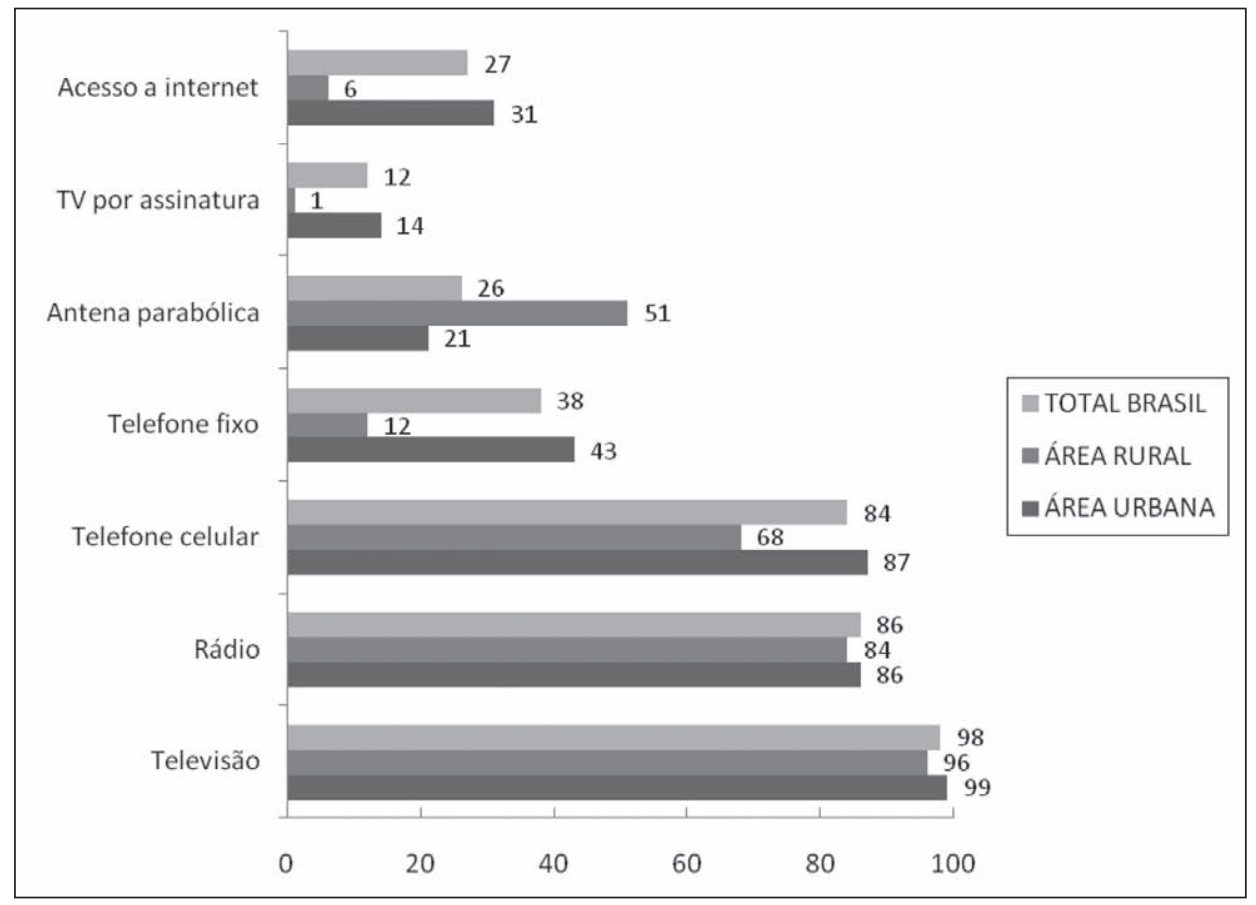

Fonte: Centro de Estudos sobre Tecnologias da Informação (Cetic.br). NIC.br - set/nov 2010.

Observaçóes: Base ponderada de 23.107 domicílios para variável de acesso a Internet e 19.998 (demais equipamentos TIC). Respostas múltiplas e estimuladas. Considerou-se que ao menos um membro do domicílio possua telefone celular.

com articulação a partir da atenção básica aos meios de diagnóstico e atenção especializada para ampliar o acesso ao sistema de saúde.

A telessaúde surge no país de maneira fragmentada, no final dos anos oitenta (SABBATINI, 1988) em universidades públicas e centros de pesquisa, com a perspectiva de contribuir para a ampliação do acesso ao cuidado em saúde com qualidade, a despeito do local onde resida o cidadão. Exemplo disso seria a obtenção de diagnóstico após o caso clínico ter sido discutido por diferentes especialistas, procedimento ao qual não se teria acesso pelo meio presencial. Diante desse desafio, indaga-se: como a telessaúde pode contribuir para que o 
SUS avance no cumprimento dos princípios de acesso universal e integralidade na atenção à saúde? Para responder a esta questão, é necessário percorrer vários caminhos, orientados por perspectivas e matrizes de pensamentos distintos.

No levantamento bibliográfico realizado sobre o tema telessaúde, observa-se que ainda é insuficiente a produção científica voltada para inserção dos saberes e práticas de telessaúde na esfera do SUS com o enfoque da Saúde Coletiva. A literatura produzida a partir das experiências de Telessaúde no Brasil, em sua grande maioria, vincula-se ao pensamento da Clínica. Esta constatação fica mais evidente ao se buscar produção científica dedicada ao tema da avaliação de iniciativas de telessaúde. O estudo demarcou esta limitação como uma das lacunas a serem superadas.

Este estudo tem como objetivo analisar o processo de inserção da telessaúde na agenda política de saúde no Brasil, a fim de agregar esforços no enfrentamento desta limitação. Para tal, vincula a busca de possíveis respostas ao campo da Saúde Coletiva, com o aporte de saberes e práticas que fundamentam a avaliação da atenção à saúde, investigando a primeira iniciativa nacional de telessaúde: a Rede Universitária de Telemedicina (Rute/RNP).

Em 1989, o Ministério da Ciência, Tecnologia e Inovação (MCT) criou a Rede Nacional de Ensino e Pesquisa (RNP), com o objetivo de construir uma infraestrutura de rede nacional de telecomunicação para a comunidade acadêmica. A Rede começou a ser montada em 1991, voltada para comunicação científica e tecnológica (CUNHA; MCCARTHY, 2006). Em 2000, a RNP foi modelada pelo Governo Federal na forma de organização social, mediante contrato de gestão com o MCT, e se estabeleceu parceria com o Centro de Pesquisa e Desenvolvimento da Telebrás $(\mathrm{CPqD})$, a fim de incentivar a pesquisa e o desenvolvimento em telecomunicações.

Em 2005, a RNP criou a Rede Universitária de Telemedicina (Rute/RNP), para implantar infraestrutura de interconexão nos hospitais universitários (HUs) e unidades de ensino de saúde no Brasil (SILVA; AMORIM, 2009). A Rute/RNP apoiou iniciativas de telessaúde, na época incipientes, ao facilitar o intercâmbio entre grupos de pesquisas, por rede de alta velocidade. Assim, em meados de 2000, a telessaúde floresceu em hospitais universitários públicos, como estratégia 
intersetorial de Estado vinculada ao MCT, envolvendo os Ministérios da Saúde (MS) e da Educação, sendo fomentada pela Rute/RNP.

A partir de 2007, o MS implementou o Programa Nacional de Telessaúde, coordenando iniciativas em nove estados. Em 2010, em articulação com a Rute/ RNP, o Programa Nacional de Telessaúde ${ }^{2}$ oficializou as ações de teleducação e teleassistência ${ }^{3}$. Em 2011, o programa foi renomeado para Telessaúde Brasil Redes.

A Rute/RNP tem congregado instituições públicas de pesquisa e inovação dedicadas à telessaúde e apoia operacionalmente grupos multi-institucionais de discussão, os Special Interest Groups (SIGs), atividade nucleadora da rede (COURY et al., 2010). A fase inicial da Rute/RNP (2006-2008) foi composta por projetos de 12 universidades federais, três universidades estaduais (Universidade de São Paulo com dois projetos), uma organização de direito privado sem fins lucrativos, um hospital estadual e uma fundação pública federal, contemplando, ao todo, grupos docentes universitários em 13 estados. Em cinco (MG, PE, SC, RJ, SP) dos nove estados que compuseram o projeto piloto do Telessaúde Brasil Redes/ $\mathrm{MS}^{4}$, a equipe Rute/RNP atuou nas duas iniciativas (figura 1).

Participam dessa etapa 19 instituições na qualidade de centros de referência em atendimento clínico e lócus da pesquisa translacional ${ }^{5}$ em saúde. Desse conjunto, dez também formam a composição inicial da Rede Nacional de Pesquisa Clínica ${ }^{6}$. $\mathrm{Na}$ história recente brasileira, cabe destacar a tendência de articulação dos grupos que desenvolvem pesquisa translacional nos HUs públicos com pesquisa e inovação na Tecnologia de Informação em Saúde (TIS), protagonizados por pesquisadores e profissionais atuantes na Rute/RNP. Essa dinâmica propicia oportunidades de circulação de saberes oriundos de diferentes abordagens, mas a contribuição das práticas e saberes ancorados na Saúde Coletiva ainda se apresenta residual. A situação se agrava quando há necessidade de processo avaliativo das iniciativas de telessaúde.

Diante dessa constatação, urge refletir sobre as possibilidades de aporte teórico das ciências sociais e humanas, do planejamento de serviços de saúde e da epidemiologia enquanto saberes constitutivos da Saúde Coletiva, na avaliação das iniciativas de telessaúde, em diferentes dimensões, como, por exemplo, sua inserção na agenda política de saúde no país. 


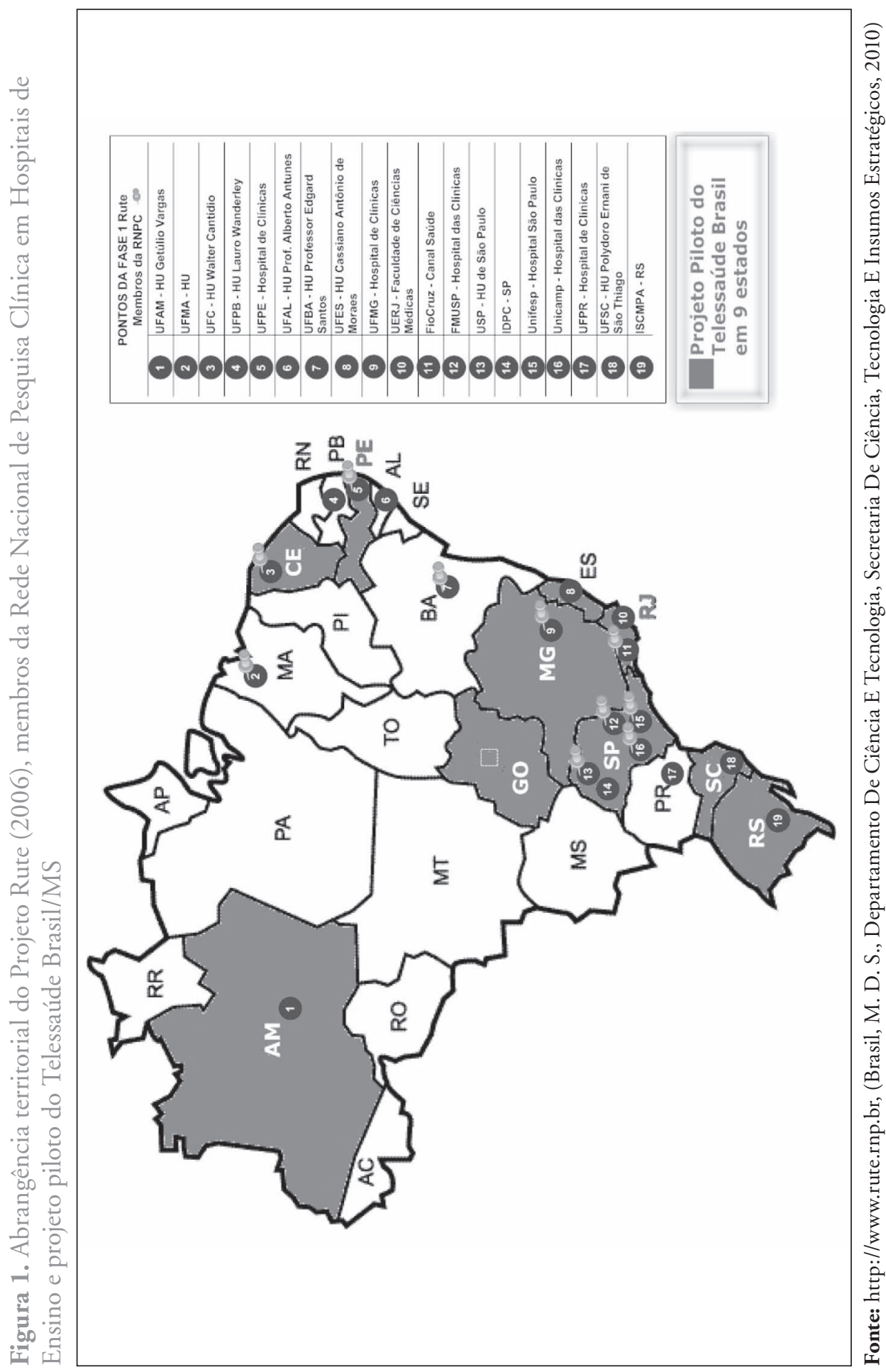


Segundo Sayão (2001), modelo é criação cultural que pretende representar aspectos da realidade, a fim de torná-los descritíveis qualitativa e quantitativamente e, em alguns casos, observáveis. Modelo teórico é um sistema hipotético-dedutivo que se refere a um objeto-modelo, sendo este uma representação conceitual esquemática de dada situação real ou suposta como tal (MEDINA et al., 2005). A construção, adoção e rejeição de modelos estão presentes na Saúde Coletiva como campo articulado de diversas práticas e saberes (CAMPOS, 2000). A partir dessa visão, realiza-se levantamento de modelos teóricos de avaliação largamente utilizados no campo da saúde, em especial da Saúde Coletiva, que permitam, ao longo desta análise, refletir sobre o "nascimento da telessaúde" no Brasil. Dá-se destaque para a experiência da Rute/RNP, no período de 2005, ano de sua criação pela RNP/MCT, a 2011.

Para tal, opta-se pela aplicação de dois modelos tradicionalmente usados na avaliação em saúde: o modelo lógico (MEDINA et al., 2005) utilizado em avaliação de programas de saúde, por sua ampla capacidade descritiva do objeto que está sendo avaliado, considerando a Rute/RNP como programa. Já o modelo de múltiplos fluxos (KINGDON, 2002) de análise de políticas públicas é escolhido por sua capacidade de identificação de atores na emergência de políticas, considerando a telessaúde como política do SUS.

O desenho do modelo lógico tem sido a primeira prática no planejamento de avaliação de programas (SILVA, 2005). Sua aplicação é realizada a partir da coleta e análise crítica dos documentos elaborados em 2005 para a composição da Rute/RNP. A partir dessas fontes, o modelo lógico proposto procura explicitar componentes essenciais e secundários do programa, assim como seus efeitos.

O modelo de múltiplos fluxos visa retratar como as agendas governamentais são formadas e alteradas, além de atribuir destaque à dinâmica das ideias no processo político. Ele procura esclarecer o motivo pelo qual alguns problemas se tornam importantes de uma hora para outra e como uma ideia emerge, transformando-se em política pública.

No caso da telessaúde e do campo de observação selecionado para a pesquisa, os 19 projetos iniciais ${ }^{7}$ da Rute/RNP (2006-2008), aborda-se um conjunto de interlocutores que representa importante comunidade política de especialistas, com diferentes lugares de fala ${ }^{8}$ e papéis. Trata-se de professores/pesquisadores que, 
ao mesmo tempo, formam profissionais de saúde, coordenam projetos de gestão e pesquisa e, na maioria das vezes, legitimam seu discurso na evidência científica.

O modelo de múltiplos fluxos de análise de políticas, conhecido como modelo de Kingdon, pertence à corrente norte-americana inaugurada por Lasswell (1956): pesquisas com metodologia indutiva, de cunho descritivo, denominados estudos de policy-making (REGONINI, 2001). Cuida-se de abordagem baseada em problemas em dada sequência de estágios: identificação do problema, enumeração dos objetivos, formulação e escolha da solução (SOUZA, 2007). Uma das críticas a essa corrente é que poucas soluções são examinadas, envolvendo pequenas mudanças. A variedade dos policy studies é composta por pesquisas com caráter prescritivo ou com metodologias hipotético-dedutivas, além de correntes pós-positivistas, argumentativas e pragmáticas (FARIA, 2003).

Kingdon analisa a complexidade da formação da agenda política governamental nas anarquias organizadas (COHEN et al., 2011). Tem-se por anarquias organizadas instituições que trabalham em condições de grande incerteza e ambiguidade, tais como universidades e governos nacionais, que têm objetivos diversos do lucro comercial e, muitas vezes, vagos e compartilhados internamente. Há três principais características nas instituições que são consideradas anarquias organizadas no que diz respeito às decisões: preferência problemática, tecnologia pouco clara e participação governamental fluída.

O modelo de múltiplos fluxos é elaborado por Kingdon para a análise de processos definidores da agenda e determinantes de políticas justamente em setores de anarquias organizadas. Sua pesquisa é baseada em entrevistas com gestores do setor saúde e de transportes (1976-1979) associada a estudo de casos e levantamentos. Ele privilegia o processo de formação e execução das políticas, analisando desde o reconhecimento do problema, passando pelo contexto político, à elaboração e ao refinamento das ideias para resolver este problema e à ação propriamente dita da política pública. $\mathrm{O}$ foco está nos empreendedores de políticas, bem como nas comunidades políticas, atuando nas janelas de oportunidades encontradas para afirmar seus interesses.

A aplicação do modelo teórico de múltiplos fluxos de política pública para analisar o surgimento da telessaúde no cenário político nacional é baseada na observação, participação e análise de atas de reuniōes do Comitê Assessor da Rute/RNP, da Comissão Especial de Estudo em Informática em Saúde da 
Associação Brasileira de Normas Técnicas (ABNT), da legislação pertinente, entre outras reuniōes e congressos científicos sobre o tema. O período estudado compreende 2009 a 2011.

Três são os fluxos iniciais para a inclusão de dada questão na agenda pública (KINGDON, 2002, p. 87):

- Do problema;

- Das alternativas - propostas de políticas públicas; e

- Do processo político.

Os fluxos do problema, das alternativas e do processo político são independentes. Acontecem paralelos, mas não necessariamente sincrônicos. Em momentos críticos, esses três fluxos convergem e nessas ocasiōes são produzidas mudanças na agenda, em que se abrem as janelas de oportunidade.

No fluxo de problemas se encontram os indicadores, a visibilidade dos eventos e o clamor nacional sobre determinada questão, segundo os formuladores de políticas. Questões são todos os fatos que afetam a coletividade e, ao mesmo tempo, são percebidas pela gestão. As questões só se tornam problemas quando os formuladores de políticas entendem o fato como uma situação sobre a qual podem fazer algo a respeito. Neste ponto, o modelo é fundamental porque tudo depende de como o formulador de políticas interpreta e percebe a questão para que ela vire um problema.

Nesse modelo, investiga-se o modo como um conjunto de soluções/ alternativas é apresentado ao formulador. Em alguns casos, ele tem acesso a um conjunto de soluções e posteriormente identificará o problema. Para explicar essa característica da anarquia organizada, Kingdon resgata o processo biológico de seleção natural, exemplificando o caso das moléculas que flutuam no caldo primitivo. $\mathrm{O}$ autor entende que ideias são geradas nas comunidades de políticas e flutuam em um caldo primitivo de políticas. Nele, algumas ideias permanecem intactas, outras se confrontam e combinam, transformando-se em novas propostas. As ideias que sobrevivem são aquelas que são viáveis do ponto de vista técnico e que têm custos toleráveis.

Há uma terceira condição: elas tocam em crenças compartilhadas com o público em geral e também sensibilizam os formuladores de políticas. Em outras palavras, a análise de custo-efetividade ou o indicador epidemiológico não 
podem ferir o conjunto de valores partilhados por esta sociedade para que uma alternativa, solução ou ideia seja aceita e se torne uma política a ser implementada.

Capella (2007) lista componentes dessas comunidades geradoras de políticas, em exercício de aproximação à realidade brasileira. São pesquisadores, assessores parlamentares, acadêmicos, funcionários públicos, analistas membros de grupos de interesses, conglomerados de empresas etc.

No fluxo das alternativas, as propostas são levantadas e aperfeiçoadas via educação, ensaios e rumores. Trata-se de fluxo em que critérios de seleção das soluções são expostos, tais como viabilidade técnica e valores agregados. Kingdon concluiu que este fluxo tem como fator decisório a popularidade e fama política.

O fluxo do processo político revela o clamor nacional, acordos percebidos e rotação de pessoas na esfera burocrática. Neste fluxo, barganha-se para construir consensos, aparecem disposições comerciais de apoio, coligaçôes e os atores se comprometem a fim de ganhar aceitação. Para entender o modelo, é preciso partir da premissa de que os problemas são construçôes sociais, envolvendo interpretação (CAPELLA, 2007). Por isso, não é possível simplesmente inferir que os três fluxos se combinam e que, a partir daí, cria-se uma prospecção. Nesse sentido, é possível identificar, no modelo, a valorização do discurso, da linguagem, assim como a possibilidade de abordagem qualitativa para investigar a emersão de políticas.

Cabe destacar que este estudo procura se alinhar ao estabelecido no Programa de Avaliação para Qualificação concebido em 2011 pelo Ministério da Saúde. Sua finalidade está em avaliar o desempenho do SUS, com prioridade aos aspectos essenciais da gestão e da atenção, na perspectiva da integralidade da atenção à saúde.

Esse Programa preconiza que a avaliação contemple ações e serviços de vigilância à saúde (promoção e prevenção); atenção básica; atenção ambulatorial e hospitalar de média e alta complexidade; e atenção às urgências, emergências e assistência farmacêutica (VIACAVA et al., 2011). Assim, serviços de telessaúde emergentes também estarão sob esse crivo: não só para garantir acesso ao sistema a despeito da localidade onde resida o cidadão, mas também para trazer nesta suposta garantia de acesso seus diferenciais para qualificar o SUS.

\section{Resultados}

A aplicação do modelo lógico, para a análise do processo de inserção da Rute/RNP na agenda política de saúde no Brasil, evidencia limitações, efeitos específicos e avanços para o SUS em sua implantação. 


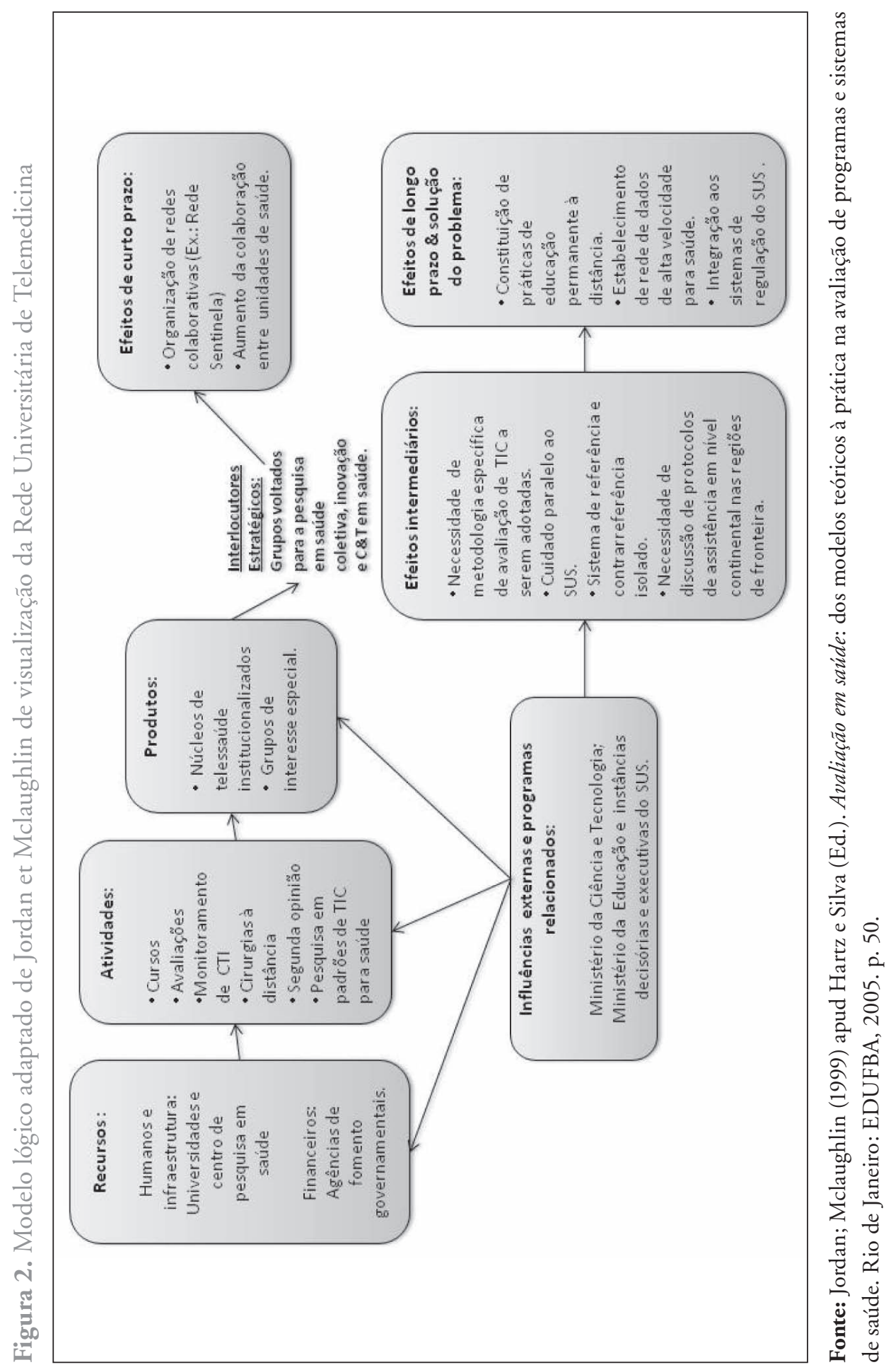

1221

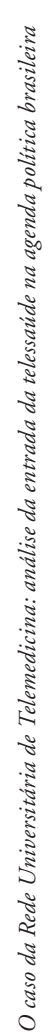


A representação visual da Rute/RNP, analisada como programa à luz do modelo lógico (figura 2), suscita questões imediatas e de longo prazo que não podem deixar de ser assinaladas em um processo de avaliação que subsidie o direcionamento de ações de telessaúde, voltadas para o aperfeiçoamento do SUS.

Dentre os resultados extraídos da análise, destacam-se pontos positivos relacionados à forma de funcionamento e dinâmica da Rute/RNP. Evidenciase sua capacidade de autoorganização e de fortalecimento de redes colaborativas diversas, como por exemplo, a Rede Sentinela no âmbito da Agência Nacional de Vigilância Sanitária ${ }^{10}$ e o aumento da colaboração entre unidades de saúde e centros de pesquisa. Essa dinâmica, voltada para o estabelecimento de interlocuções entre diferentes iniciativas, tem potencializado a discussão de protocolos de assistência, que envolvem o continente latino-americano, principalmente nas regiões de fronteira.

Como ponto negativo observado a partir da aplicação desse modelo, destaca-se a incipiente organização de uma rede de cuidado à saúde exclusivamente articulada ao ensino e à pesquisa desenvolvidos nos HUs. Essa frágil interação com o sistema pode estar relacionada à constatação de que as iniciativas de telessaúde/Rute/ RNP não têm nem interface sistêmica com a rede exclusivamente assistencial, nem conexão operacional com os sistemas de informação do SUS. Essas observações representam um alerta para policy makers. Se não houver mudança dessa tendência, ampliam-se os riscos de a telessaúde na Rute/RNP, fortemente ancorada nas instituições de ensino e pesquisa, estruturar-se de forma paralela à rede de cuidados à saúde. Uma das resultantes desse processo pode ser a geração de fluxos de referência e contrarreferência de pacientes isolados e fragmentados.

Dessa análise da aplicação do modelo lógico, depreende-se que outras demandas específicas para o SUS ganharam maior destaque com a implantação da Rute/RNP:

- a constituição de práticas de educação permanente à distância, por consenso de diferentes instâncias organizacionais, as quais se articulam em rede. Uma das questões imediatas, suscitadas a partir dessa rede, é a definição sobre "quem" e "como" certifica o saber gerado nessas práticas virtuais do ciberespaço, que antes não existiam; e

- a necessidade de estabelecer o direito de propriedade intelectual da memória produzida pelos SIGs e propagada pela Internet, que tem se tornado tema de debates éticos e políticos da telessaúde (REZENDE et al., 2010). 
Enfim, a implementação da Rute/RNP tem reforçado a necessidade de estabelecer a inclusão digital das equipes dos profissionais de saúde, que é mais do que estabelecer infraestrutura em rede de dados de alta velocidade ou apenas estar habilitado na técnica de manejar a máquina (SILVA et al., 2005). A inclusão digital é desafio intersetorial, que inclui a dimensão educacional e política (MORAES et al., 2009).

Em relação ao segundo modelo - múltiplos fluxos -, sua aplicação resultou em uma síntese de como a telessaúde emerge para uma "agenda decisória" no Brasil (figura 3). O modelo não esgota o tema, mas mostra a complexidade da emergência de uma política pública e a articulação de alguns atores estratégicos para a implantação da telessaúde no SUS.

Figura 3. Modelo de múltiplos fluxos da política pública de telessaúde no contexto brasileiro.

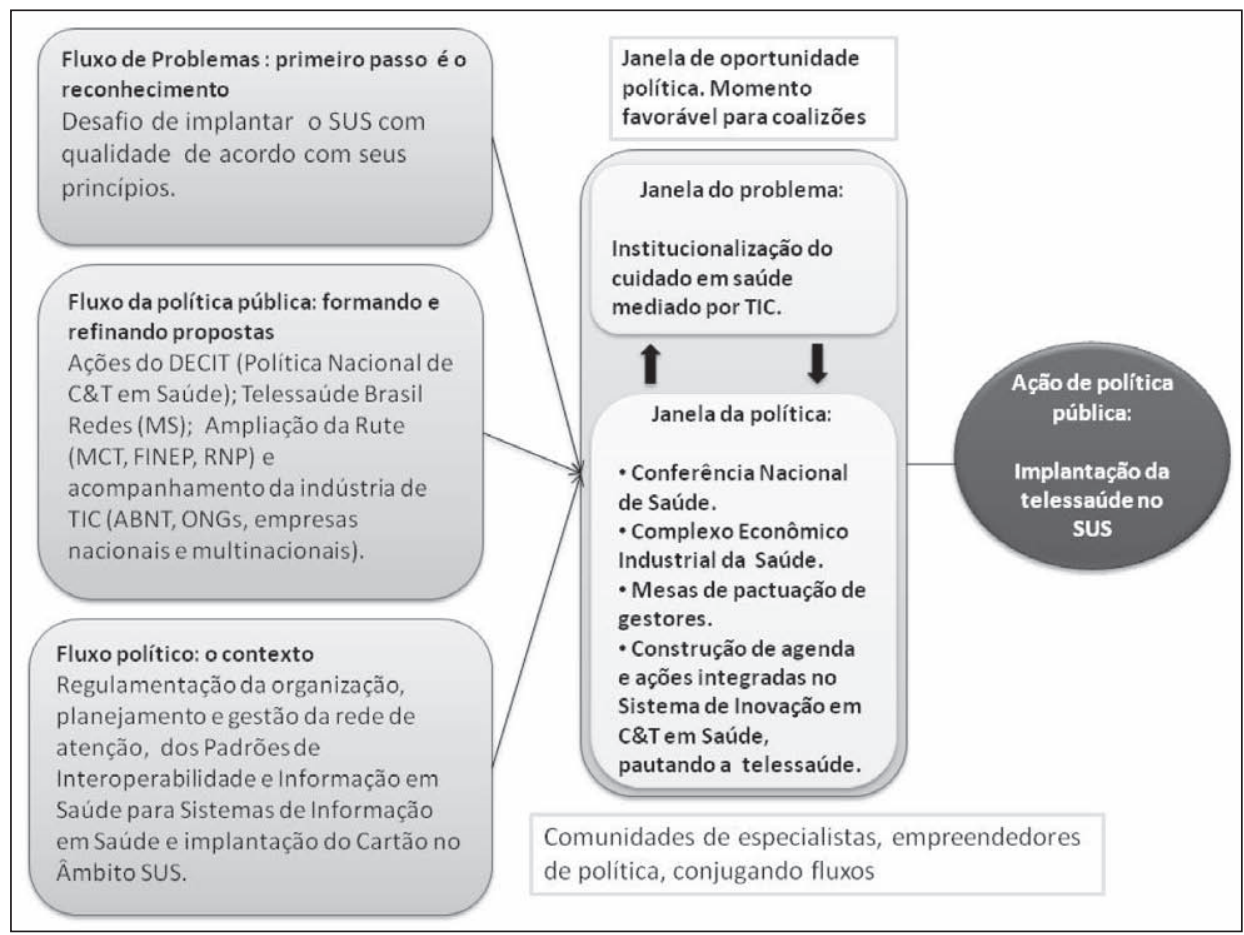

Fonte: Kingdon, J. W. Agendas, Alternatives, and Public Policies. 2. ed. New York: Longman, 2002. 
Há três mecanismos básicos que despertam interesse dos gestores, para que a questão se transforme em problema: indicadores (sistemas de informação em saúde e indicadores socioeconômicos), um conjunto de eventos, crises e símbolos (opiniāo e comoção pública) e o feedback das ações governamentais (avaliações tecnológicas e de custo-efetividade).

Quanto aos indicadores, no caso da telessaúde no Brasil, além dos sistemas de informação que evidenciam os problemas de saúde da população que demandam respostas das autoridades sanitárias, como a qualidade e o desempenho da rede de atenção, a inclusão digital emerge como uma necessidade, em especial nos setores públicos de educação e saúde. Afinal, com a conexão dos centros de pesquisa e HUs, contemplados pela Rute, em redes de alta velocidade da RNP, a ampliação da "competência" digital de suas equipes otimiza o uso da infraestrutura instalada.

Já no conjunto de eventos, crises e símbolos, uma questão relevante para a opinião pública é a teleconsulta. A primeira regulação governamental na área de telessaúde, promovida pelo Telessaúde Brasil Redes/MS, é sobre segunda opinião formativa, ${ }^{11}$ que prevê também a teleconsultoria. ${ }^{12} \mathrm{O}$ debate do ato médico sobre quem faz a consulta à distância, se tem de haver dois profissionais de saúde nas pontas, é o que mais aparece na mídia e na legislação, ${ }^{13}$ realçado como consequência da expansão da telessaúde. Daí ser necessária a regulação nesse processo.

Outros pontos a serem ressaltados como valores simbólicos são a presença crescente das TIC na vida cotidiana da população e o respectivo caráter de modernidade que o governo alcança junto à opinião pública ao implantar estas soluções informatizadas. Aspecto relevante é a ampliação do mercado e da articulação do complexo econômico-industrial da saúde com as TIC, que estimula sistemas diversos de teleconsulta e outros dispositivos eletrônicos ligados às atividades de telessaúde.

No feedback das ações governamentais, o subfinanciamento do SUS e a proliferação de avaliações econômicas sobre experiências de telessaúde, que podem desonerar a assistência à saúde, chamam a atenção dos gestores. A isso se associa a expansão do pensamento de abordar saúde como mercadoria no processo econômico-produtivo do país. 
A aplicação do modelo no fluxo, em que se observa o processo de construção

do conjunto de alternativas e soluções para o problema, resulta na identificação dos seguintes pontos:

- Incipiente discussão sobre telessaúde no âmbito do Departamento de Ciência e Tecnologia do MS, bem como da Política Nacional de Ciência e Tecnologia (C\&T) em Saúde;

- Fortalecimento do debate da incorporação da telessaúde ao SUS por intermédio das ações do Telessaúde Brasil Redes (MS);

- Suporte adicional pelas agências de fomento da política de ampliação da Rute/RNP;

- Aumento do interesse em torno de normas técnicas institucionais referentes à telessaúde por parte da indústria de TIC, representada pela ABNT, ONGs, empresas nacionais e multinacionais, fazendo interface com a Administração Pública, em especial o Departamento de Informática do SUS (DATASUS) e a Agência Nacional de Saúde Suplementar.

No fluxo do processo político, é possível observar entrepreneurs, legislação e fóruns recém-criados, em que o tema recorrente da interoperabilidade das aplicações em rede, destinadas à saúde, tem surgido quando se discute o desenho da telessaúde a ser introduzida no modelo de atenção. A análise da conjuntura política, em que se inserem a Rute/RNP e a telessaúde, evidencia a luta de interesses, dentre os quais se destacam os relacionados aos padrões de interoperabilidade para sistemas de informação em saúde e implantação do Cartão Nacional de Saúde/Registro Eletrônico de Saúde no SUS.

\section{Discussão}

Dados de 2010 relacionados ao uso de produtos e serviços de TIC dão conta de grandes disparidades regionais (quadro 2). O acesso à internet local, à telefonia, à TV interativa e segmentada, meios eletrônicos fundamentais para realizar ações de telessaúde, ainda não tem extensão territorial correspondente às necessidades do SUS. 


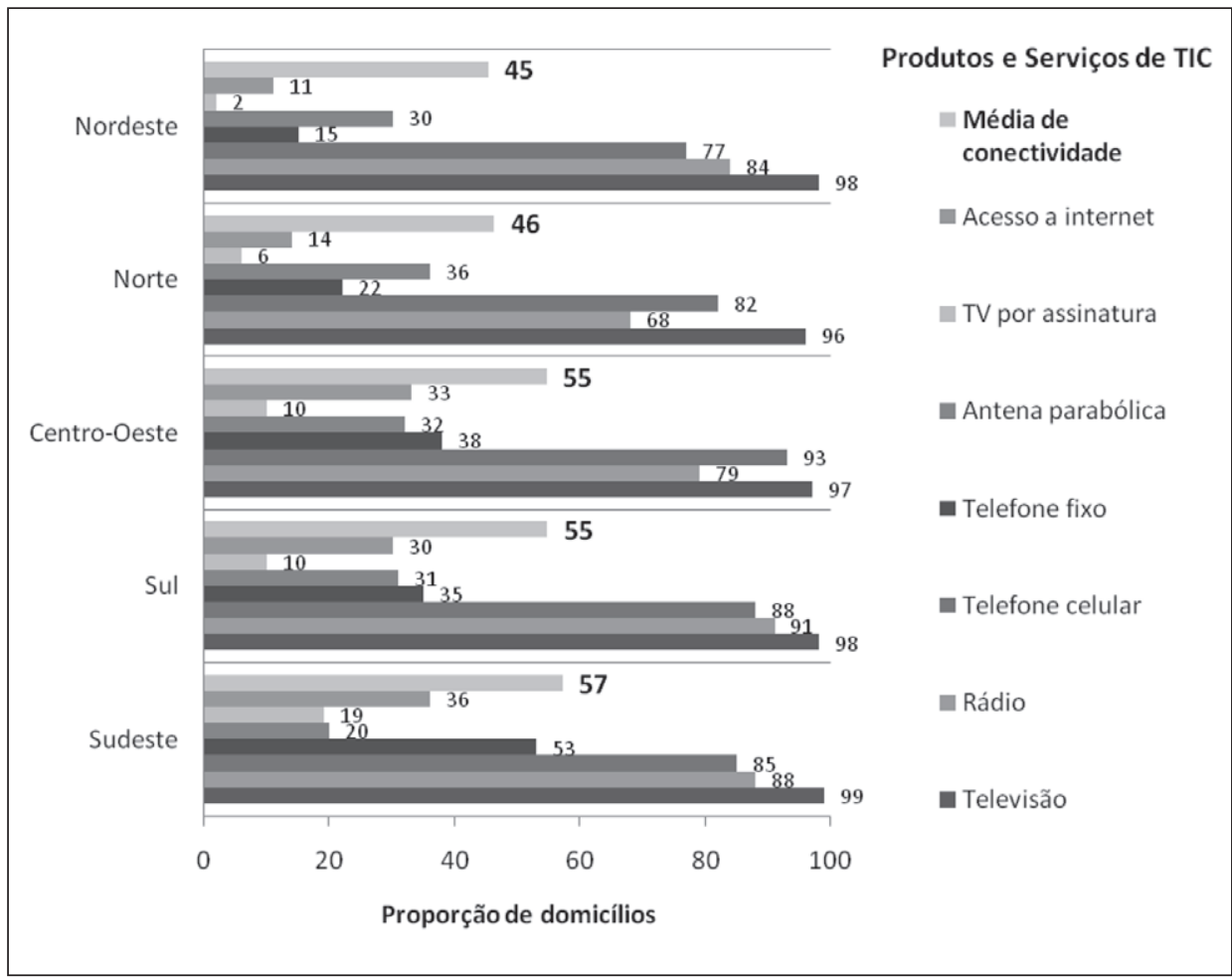

Fonte: Centro de Estudos sobre Tecnologias da Informação (Cetic.br). 2011. NIC.br - set/nov 2010 Observaçôes: A média de conectividade foi calculada através da média aritmética das variáveis apresentadas (televisão, rádio, telefone celular, fixo, antena parabólica, tv por assinatura e acesso a internet).

Nascimento (2008), ao documentar a política pública de telecomunicações, aponta que, após 30 anos (1960-1990) de investimento do Ministério das Comunicaçôes e do Sistema Telebrás em pesquisa e desenvolvimento, o Governo federal, sob o argumento de atender às demandas da globalização e da sociedade da informação, editou a Lei Geral das Telecomunicações e criou a Agência Nacional de Telecomunicações (Lei no 9.742/1997), privatizou o sistema de interconexão de dados públicos e transformou o $\mathrm{CPqD}$ em fundação de direito privado.

No contexto de um mercado privado de TIC em expansão e de demanda da sociedade por maior transparência das ações governamentais, impulsionando a expansão de ações de governo eletrônico (e-gov), a necessidade da interoperabilidade 
entre sistemas de informações, aplicativos e plataformas ganha relevância. A interoperabilidade éfundamental para ampliar o escopo de aplicações de telessaúde. O Governo federal (BRASIL, 2010) adotou o conceito de interoperabilidade da International Standard Organization ${ }^{14}$, que se refere à "habilidade de dois ou mais sistemas (computadores, meios de comunicação, redes, software e outros componentes de tecnologia da informação) de interagir e intercambiar dados de acordo com um método definido, de forma a obter os resultados esperados".

Oostveen (2010), ao refletir sobre a luta dos movimentos sociais na Holanda contra a terceirização do sistema de votação por internet após 20 anos de funcionamento, apresenta questôes relevantes sobre processo decisório e implantação de políticas públicas relacionadas às TIC, que instrumentalizam uma análise sobre a questão da interoperabilidade na saúde.

De acordo com Gauld e Goldfinch (2006, apud OOSTVEEN, 2010), os governos estão empenhados em altos investimentos nas soluções de TIC por razões variadas. Eles tendem a uma paixão tecnológica, em que servidores públicos e políticos creem que as TIC podem transformar a gerência governamental. Outro ponto ressaltado é que gestores públicos têm de lidar com uma espécie de culto ao tecnoluxo, descrito por Gauld e Goldfinch (2006, p. 18) como o entusiasmo, fingido ou real, que representantes de vendas e outros colaboradores desenvolvem por produtos e habilidades de suas empresas.

No Brasil há, como exemplo, o Projeto Piloto do Cartão Nacional de Saúde do SUS, que começou por uma licitação internacional, em 1999, para consolidar demanda política-institucional, explicitada na NOB-SUS de 1996. Magalhães (2010), em estudo sobre essa experiência, constata falta de transparência e apresenta essa característica do Projeto como uma das explicações para sua não finalização. Na década 2000-2011, gastam-se, com a promessa da integração digital do SUS com interoperabilidade, mais de duzentos milhões de dólares, sem acompanhamento do controle social (VASCONCELLOS; MORAES, 2005), acima do que se havia se destinado à cobertura vacinal da população e à reinserção de jovens infratores, somados no mesmo período (SILVA, 2007).

Tem-se, em 2010, a discussão na Comissão Intergestores Tripartite sobre a regulamentação do uso de padrōes de interoperabilidade para troca de informações entre os sistemas de informação em saúde, que acaba por gerar minuta de portaria. ${ }^{15}$ Em sua versão final, após consulta pública eletrônica, é publicada a Portaria/GM/ 
1228 MS n ${ }^{\circ} 2.073 / 2011^{16}$, que descreve 15 padrōes de informação. A análise desse documento evidencia redação confusa, que permite observar o jogo de interesses entre especialistas, empreendedores de políticas e empresas de TIC.

A tecnofilia dos desenvolvedores de sistemas tem papel importante na persuasão. O "mito do conserto tecnológico" (OOSTVEEN, 2010), entendido pelo uso agregado de tecnologias mais avançadas para resolver qualquer problema prático, influencia os formuladores de políticas. Nesse sentido, um dos parágrafos iniciais da Portaria já revela o mito do conserto tecnológico: "Considerando a racionalização e a interoperabilidade tecnológica dos serviços nos diferentes níveis da Federação para permitir o intercâmbio das informações e a agilização dos procedimentos".

O poder dessa regulamentação pode gerar prejuízos, sem o controle social esclarecido, quando analisado sob a perspectiva cibercultural, em que tecnologias computacionais se tornam ubíquas, o espaço e a vigilância se tornam líquidas ${ }^{17} \mathrm{e}$ a privacidade pode ser graduada segundo o capital social do indivíduo (LEMOS, 2010). Os serviços de telessaúde usarão intensivamente padrões para rastrear e controlar indivíduos em nome de suas respectivas qualidades de vida. Mas, como indaga Moraes (2002): "quem vigiará o vigilante?”

Identifica-se como problema a necessidade de definição de regras claras sobre a telessaúde no SUS, dado que nela há uma tensão entre interesses públicos, coletivos e indústria privada. Em qualquer lugar é possível fazer telessaúde para os ricos, enquanto os pobres terão seus corpos esquadrinhados, se não houver um processo democrático emancipador (MORAES, 2002) em relação à implantação de qualquer solução de tecnologia da informação para a saúde. Portanto, como janela de oportunidade ainda pouco explorada nessa problemática, há a possibilidade de construção de uma pauta de ações integradas em telessaúde e interoperabilidade de sistemas de informação do SUS, no âmbito do Sistema de Inovação em C\&T em Saúde.

Essa pauta pode estimular uma ampla discussão com a sociedade e, ao mesmo tempo, tornar as demandas específicas das iniciativas de telessaúde parte indissociável do complexo econômico-industrial da saúde. Um dos dispositivos empregados para inovações em saúde é a Lei de Incentivo à Inovação e à Pesquisa Científica e Tecnológica (Lei no 10.973/2004), que deveria ser usada intensamente pelas atividades de telessaúde do SUS. 
As três características da anarquia organizada em relação às decisões preferência problemática, tecnologia pouco clara e participação governamental fluída - estão presentes no debate de telessaúde no Brasil no período 2005-2011, conforme evidenciado ao longo desta análise.

As preferências problemáticas estão presentes no atual cenário. É possível que se opte, de maneira rápida e não transparente, por "soluções fechadas", com pouca ou nenhuma interoperabilidade, na expectativa de agilizar a assistência à saúde. Com isso, os gestores acreditam satisfazer o clamor nacional pelo fim das filas nos estabelecimentos de saúde. Porém, em curto prazo, essa decisão tem a possibilidade de gerar processo de dependência/aprisionamento a empresas, plataformas ou sistemas (MORAES; GÓMEZ, 2007), que pode colapsar o sistema de referência e contrarreferência das redes públicas de atenção e tornar inviável economicamente o SUS.

É preciso que a administração pública, em suas diferentes esferas inclusive produtivas, esteja ciente das vantagens e limitações das tecnologias empregadas. De igual modo, é preciso que os contratos prevejam a transferência tecnológica desses sistemas de informação em saúde para o Estado, pois estes são acima de tudo um bem público (MORAES, 2002).

A participação governamental fluida se manifesta pelo incipiente acompanhamento institucional da telessaúde no SUS por parte do Governo federal. A legislação atual do MS coloca as ações de telessaúde sob a responsabilidade das Secretarias de Gestão do Trabalho e Educação na Saúde e de Atenção à Saúde, a quem cabe definir alguns padrões de gestão, monitoramento e avaliação em telessaúde. Os tipos de serviços de telessaúde destacados nesse arcabouço institucional são teleconsultoria, tele-educação, segunda opinião formativa e telediagnóstico.

Na organização do Telessaúde Brasil Redes/MS, há uma clara separação entre pesquisa e assistência, criando núcleos técnicos científicos, que fornecem os serviços, e pontos ou unidades de telessaúde, que solicitam os serviços de apoio à assistência aos núcleos. Um avanço importante, identificado no período de 2005-2011, é a integração dos núcleos e pontos ao Sistema de Cadastro Nacional de Estabelecimentos de Saúde (SCNES). No entanto, a parceria entre Telessaúde Brasil Redes e Rute/RNP, que estava clara em 2010, agora faz parte de uma Portaria revogada (Portaria GM/MS no 402/2010). A legislação 
atual é confusa, na medida em que cria outra instância para definir o padrão tecnológico de interoperabilidade, que viabilizará a operação do Telessaúde Brasil Redes. Essa realidade suscita dúvidas, dentre as quais convém destacar a indagação de como ficarão os projetos e a inovação em telessaúde no escopo da Rute/RNP nesse contexto.

A expectativa é a de que os resultados alcançados, a partir desta análise, contribuam para futuros estudos dedicados à compreensão de limitações e avanços da telessaúde no país. A telessaúde, no âmbito do SUS, precisa ser apropriada para além do nicho de negócio. Ela constitui processo de mudança cultural e poderosa ferramenta de integração entre a inovação e a saúde pública, com vistas a alcançar a almejada integralidade na atenção. ${ }^{18}$

\section{Referências}

BECK, U. O que é globalização? 1. São Paulo: Paz e Terra, 1999.

BRAGA, J.L. Lugar de fala: como conceito metodológico no estudo de produtos culturais. In: ENCONTRO NACIONAL DA COMPÓS, 5. COMPÓS. São Paulo. I: 19 p. 1997.

BRASIL, MPOG. Panorama da interoperabilidade no Brasil. INFORMAÇÃO, S. D. L. E. T. D. Brasília, DF: MP/SLTI: 251 p. 2010.

CAMPOS, G. W.D.S. Saúde pública e saúde coletiva: campo e núcleo de saberes e práticas. Ciência \& Saúde Coletiva, v. 5, p. 219-230, 2000. Disponível em: < http://www.scielo.br/ scielo.php?script $=$ sci_arttext $\&$ pid $=S 1413-81232000000200002 \& n r m=i s o ~>$. Acesso em: 18 fev. 2012.

CAPELLA, A. C. N. Perspectivas Teóricas sobre o Processo de formulação de Políticas Públicas. In: HOCHMAN, G.; ARRETCHE, M.T.S. et al (Ed.). Políticas públicas no Brasil. Rio de Janeiro: Fiocruz, v.1, 2007. p.87-124.

COHEN, M.D. et al. A garbage can model of organizational choice. Gestion Y Politica Publica, v. 20, n. 2, p. 247-290, 2011. Disponível em: < http://www.jstor.org/stable/2392088>. Acesso em: 14 mar. 2012.

COURY, W.B. et al. Implementing RUTE's Usability - the Brazilian Telemedicine University Network. 2010. Disponível em: < http://www.objnursing.uff.br/index.php/nursing/article/ view/j.1676-4285.2010.3176 >. Acesso em: 4 dez. 2011.

CUNHA, M.B.D.; MCCARTHY, C. Estado atual das bibliotecas digitais no Brasil. In: IBICT (Ed.). Bibliotecas digitais: saberes e práticas. 2. Brasília: IBICT, 2006. p.25-54.

DE MORAES, I.H.S. et al. Digital inclusion and health counselors: A policy for the reduction of social inequalities in Brazil. Ciencia e Saude Coletiva, v. 14, n. 3, p. 879-888, 2009. 
Disponível em: < http://www.scopus.com/inward/record.url?eid=2-s2.0-68649095460\&pa rtnerID=40\&md5=fd8e6e3924b8fea8197a3532b62371f6 >. Acesso em: 20 fev. 2012.

FARIA, C.A.P.D. Idéias, conhecimento e políticas públicas: um inventário sucinto das principais vertentes analíticas recentes. Revista Brasileira de Ciências Sociais, v. 18, p. 21-30, 2003. Disponível em: < http://www.scielo.br/scielo.php?script=sci_arttext\&pid=S010269092003000100004\&nrm=iso >. Acesso em: 19 jan. 2012.

KIngDON, J. W. Agendas, Alternatives, and Public Policies. 2. New York: Longman, 2002. 280 .

LASSWELL, H.D. The decision process; seven categories of functional analysis. University of Maryland Press, 1956. 23.

LEMOS, A. Mídias locativas e vigiância. Sujeito inseguro, bolhas digitais, paredes virtuais e territórios informacionais. In: SULINA, E. (Ed.). Vigilancia e visibilidade: espaço, tecnologia e identificação. Porto Alegre: Sulina, 2010. p.296.

MAGAlHĀES, M. Desafios da Gestão de uma Base de Dados de Identificação Unívoca de Indivíduos: a experiência do Projeto Cartão Nacional de Saúde no SUS. 2010. 107 Dissertação (Mestrado em Saúde Pública) - Escola Nacional de Saúde Pública Sergio Arouca, Fundação Oswaldo Cruz, Rio de Janeiro, 2010.

MATTOS, R.A. Os Sentidos da Integralidade: algumas reflexões acerca de valores que merecem ser defendidos. In: PINHEIRO, R.; MATTOS, R.A. (Org.). Os sentidos da integralidade na atenção e no cuidado à saúde. 6a. Rio de Janeiro: Cepesc, 2006.

MEDINA, M.G. et al. Usos de modelos teóricos na avaliação em saúde: aspectos conceituais e operacionais. In: FIOCRUZ (Ed.). Avaliação em saúde: dos modelos teóricos à prática na avaliação de programas e sistemas de saúde. Rio de Janeiro: Fiocruz, 2005. p.41.

MORAES, I.H.S. Política, tecnologia e informação em saúde: a utopia da emancipação. 1. Salvador: Casa da Qualidade, 2002. 171.

MORAES, I.H.S.D.; GÓMEZ, M.N.G.D. Informação e informática em saúde: caleidoscópio contemporâneo da saúde. Ciência \& Saúde Coletiva, v. 12, p. 553-565, 2007. Disponível em: < http://www.scielo.br/scielo.php?script=sci_arttext\&amp >. Acesso em: 12 fev 2012.

NASCIMENTO, J.R. O Setor de Telecomunicaçôes: História e Políticas Públicas no Brasil. 2008. 77p. Dissertação (Mestrado Profissionalizante) - Programa de Pós-Graduação em Administração das Faculdades Ibmec, FAculdade de Economia e Finanças IBMEC, Rio de Janeiro, 2008.

OOsTVEen, A.-M. Outsourcing Democracy: Losing Control of e-Voting in the Netherlands. Policy \& Internet. London, v. 2, 2010.

PAULA, S.D.; GREGGIO, S.; DACOSTA, J.C. O uso de células-tronco na asfixia perinatal: do laboratório à prática clínica. Jornal de Pediatria, v. 86, p. 451-464, 2010. Disponível em: < http:// 
www.scielo.br/scielo.php?script=sci_arttext $\&$ pid $=$ S0021-75572010000600003\&nrm $=$ iso >. Acesso em: 23 jan. 2012.

REGONINI, G. Capire le politiche pubbliche. Bologna: il Mulino, 2001.

REZENDE, E.J.C. et al. Ethics and eHealth: Reflections for a safe practice. Revista Panamericana de Salud Publica, v. 28, n. 1, p. 58-65, 2010. Disponível em: < http://www. scopus.com/inward/record.url? eid=2-s2.0-77956823942\&partnerID = 40\&md5 = cbc4d05 bb16b1197547add9de92e7a1b >. Acesso em: 8 mar. 2012.

SABBATINI, R.M. E. Usando a telemática em saúde. Revista Brasileira de Informática em Saúde, São Paulo, v. 1, n. 6, p. 5-12, 1988. Disponível em: < http://bases.bireme.br/cgi-bin/ wxislind.exe/iah/online/list. >. Acesso em: 17 dez. 2011.

SAYÃO, L.F. Modelos teóricos em ciência da informação - abstração e método científico | Sayão | Ciência da Informação. Ciência da Informação. Brasília, v. 30, p. 82-91, 2001.

SILVA, A.B. Multimidia e Conectividade dos Conselhos de Saúde: a plenária virtual permanente. 2005. 126p. Dissertação (Mestrado em Saúde Pública) - Escola Nacional de Saúde Pública Sergio Arouca, Fundação Oswaldo Cruz, Rio de Janeiro.

SILVA, A.B.; AMORIM, A.C. A Brazilian educational experiment: Teleradiology on web TV. Journal of Telemedicine and Telecare, v. 15, n. 7, p. 373-376, 2009. Disponível em: < http://www.scopus.com/inward/record.url? eid=2-s2.0-70350126956\&partnerID =40 \&m d5 =c8cc23a7f68f6e48f7dac3f95e96759f >. Acesso em: 20 fev. 2012.

SILVA, A.S.L. Cartão Nacional de Saúde e o prontuário eletrônico do Paciente no Brasil: Uma visão do estado atual. In: SIMPÓSIO DE EXCELÊNCIA EM GESTÃO E TECNOLOGIA, 4. Rio de Janeiro: Associação Educacional Dom Bosco, 2007.

SILVA, H. et al. Inclusão digital e educação para a competência informacional: uma questão de ética e cidadania. Ciência da Informação, v. 34, p. 28-36, 2005. Disponível em: < http:// www.scielo.br/scielo.php?script $=$ sci_arttext $\&$ pid $=$ S0100-19652005000100004\&nrm $=$ iso >. Acesso em: 5 mar. 2012.

SILVA, L.M.V. Conceitos, abordagens e estratégias para a avaliação em saúde. In: HARTZ, Z.M.D.A.; SILVA, L.M.V.D. (Ed.). Avaliação em saúde: dos modelos teóricos à prática na avaliação de programas e sistemas de saúde. Rio de Janeiro: Fiocruz, 2005. p.275.

SOUZA, C. Estado da Arte da Pesquisa em Políticas Públicas. In: FIOCRUZ (Ed.). Políticas públicas no Brasil. Rio de Janeiro: Fiocruz, 2007. p.65-86.

VASCONCELlOS, M.M.; MORAES, I.H.S. National Policy on Health Information, Informatics and Communication: a Pact to be Built. Saúde em Debate. Rio de Janeiro, v. 29, p. 86-98, 2005.

VIACAVA, F. et al. PROADESS: Avaliação de Desempenho do Sistema de Saúde Brasileiro: indicadores para monitoramento. Rio de Janeiro: Fiocruz, 2011. 
${ }^{1}$ A pesquisa translacional em saúde (SCHMITTDIEL; GRUMBACH et al., 2010) é um modo de fazer pesquisa que surge como uma resposta para diminuir o gap entre a produção de inovação e seu uso efetivo na intervenção junto à população.

${ }^{2}$ Vide http://www.ufrgs.br/telessauders/arquivos\%20pdf/portaria\%20MS\%2035_04_01_07.pdf

${ }_{3}$ Vide http://www.telessaudebrasil.org.br/php/level.php?lang=pt\&component=42\&item =16

${ }^{4}$ Vide http://telessaude.bvs.br/tiki-read_article.php?articleId=357

${ }^{5}$ A pesquisa translacional pode ser definida também como um processo que parte da prática baseada em evidências em direção a soluções sustentáveis para problemas de saúde da comunidade (PAULA, GREGGIO et al., 2010).

${ }^{6}$ HU da UFMA, HU Walter Cantídio da UFC, HC da UFPE, HU Professor Edgard Santos da UFBA, HC da UFMG, Fiocruz, HC da FMUSP, HU de São Paulo da USP, Hospital São Paulo da Unifesp, HC da Unicamp. Rede Nacional de Pesquisa Clínica em Hospitais de Ensino (RNPC) se propõe ser modelo institucional de pesquisa clínica baseado nas melhores práticas de pesquisa voltadas às urgências do SUS.

${ }^{7}$ (1) Hospital Universitário Getúlio Vargas da UFAM; (2) HU da UFMA; (3) HU Walter Cantídio da UFC; (4) HU Lauro Wanderley da UFPB; (5) Hospital de Clínicas da UFPE; (6) HU Professor Alberto Antunes da UFAL; (7) HU Professor Edgard Santos da UFBA; (8) HU Cassiano Antônio de Moraes da UFES; (9) HC da UFMG; (10) Faculdade de Ciências Médicas da UERJ; (11) Canal Saúde da Fundação Oswaldo Cruz; (12) HC da FMUSP; (13) HU de São Paulo da USP; (14) Instituto Dante Pazzanese de Cardiologia; (15) Hospital São Paulo da Unifesp; (16) HC da Unicamp; (17) HC da UFPR; (18) HU Polydoro Ernani de São Thiago da UFSC; e (19) Irmandade da Santa Casa de Misericórdia de Porto Alegre.

${ }^{8}$ A expressão é usada como conceito metodológico no estudo de produtos culturais (BRAGA, 1997), que enseja na articulação entre fala, situação e textos sobre o assunto, relacionando a fala com os registros disponíveis sobre o tema estudado.

9 Vide http://portal.saude.gov.br/portal/arquivos/pdf/programa_avaliacao_qualificacao_SUS_ versao3.pdf

${ }^{10}$ Vide http://www.anvisa.gov.br/servicosaude/hsentinela/apresentacao.htm

${ }^{11}$ Segundo a Portaria GM/MS no 2.546/2011, trata-se de resposta sistematizada, construída com base em revisão bibliográfica, nas melhores evidências científicas e clínicas e no papel ordenador da atenção básica à saúde, a perguntas originadas das teleconsultorias, e selecionadas a partir de critérios de relevância e pertinência em relação às diretrizes do SUS.

${ }^{12}$ Segundo a Portaria GM/MS no 2.546/2011, teleconsultoria é consulta registrada e realizada entre trabalhadores, profissionais e gestores da área de saúde, por meio de instrumentos de telecomunicação bidirecional, com o fim de esclarecer dúvidas sobre procedimentos clínicos, ações de saúde e questôes relativas ao processo de trabalho, podendo ser síncrona ou assíncrona.

${ }^{13}$ Vide as Portarias GM/MS: no 561/2006, que institui, no âmbito do Ministério da Saúde, a Comissão Permanente de Telessaúde; no 35/2007, que institui, no âmbito do Ministério da Saúde, o Programa Nacional de Telessaúde; no 402/2010, que institui, em âmbito nacional, o Programa Telessaúde Brasil para apoio à Estratégia de Saúde da Família no Sistema Único de Saúde; e nº 2.546/ 2011, que redefine 
1234 e amplia o Programa Telessaúde Brasil, que passa a ser denominado Programa Nacional Telessaúde Brasil Redes. Quanto aos procedimentos éticos, em relação à telemedicina especificamente, o Conselho Federal de Medicina lançou três Resoluçôes importantes: a no 1.639/2002, que define "Normas Técnicas para o Uso de Sistemas Informatizados para a Guarda e Manuseio do Prontuário Médico”, a 1.643/2002 define e disciplina a prestação de serviços através da telemedicina; e a no 1.974/2011, que estabelece critérios norteadores da propaganda em Medicina, conceituando anúncios, divulgação de assuntos médicos, sensacionalismo, autopromoção e proibiçôes referentes à matéria.

${ }^{14}$ Vide relatório técnico (ISO/TR 16056-1, 2004).

${ }^{15} \mathrm{http}: / /$ portal.saude.gov.br/portal/arquivos/pdf/padroes_240910.pdf

${ }^{16}$ http://telessaude.bvs.br/tiki-download_file.php?fileId=2427

${ }^{17}$ Termo cunhado pelo filósofo Zigmunt Bauman para designar algumas características da pós-modernidade como mundo diversificado e polifônico, que mais recentemente o autor tem caracterizado pela metáfora da liquidez.

${ }^{18}$ A.B. Silva concebeu o artigo e ambas as autoras participaram da elaboração e revisão do texto. 
The case of Telemedicine University Network: analysis of telehealth entry in the Brazilian political agenda

A qualitative study on the policy design of telehealth in Brazil based on valuation models in health and public policy analysis, specifically the proposed multiple streams of analytical and logical model for evaluating health programs. Data sources consisted of documents related to the creation of Telemedicine University Network (Rute), articles available in the literature on telehealth and health evaluation, minutes of meetings and participating in various forums. The model focuses multiple streams in the early stages of the cycle of public policy: the construction of the government agenda and choice of alternative policies. We sought to delineate the composition of Rute and answer constraints which led federal managers from various sectors to recognize the quality of the system as a public health problem and the choice of implementing telehealth programs as one of the alternatives. The cases analyzed showed that in the flux convergence problems with favorable political environment, a window of opportunity opened up for the deployment of telehealth to eventually ascend as an alternative in the political agenda of the government decision. But for this, there is urgent need for digital inclusion in the territory and identify the technologies of information and communication as an innovation for SUS.

Key words: telehealth; public policies; assessment. 